\title{
Dynamical Evolution of Mass-Segregated Clusters
}

\author{
Enrico Vesperini ${ }^{1}$, Steve McMillan ${ }^{1}$ and Simon Portegies Zwart ${ }^{2}$ \\ ${ }^{1}$ Department of Physics, Drexel University, Philadelphia, PA, USA \\ ${ }^{2}$ Astronomical Institute "Anton Pannekoek" and Section Computational Science, University \\ of Amsterdam
}

\begin{abstract}
We present the results of a survey of $N$-body simulations aimed at exploring the implications of primordial mass segregation on the dynamical evolution of star clusters. We show that, in a mass-segregated cluster, the effect of early mass loss due to stellar evolution is, in general, more destructive than for an unsegregated cluster with the same density profile and leads to shorter lifetimes, a faster initial evolution toward less concentrated structure and flattening of the stellar initial mass function.
\end{abstract}

Keywords. globular clusters: general, methods: n-body simulations, stellar dynamics

\section{Introduction}

Mass segregation, the tendency of more massive stars to preferentially populate the inner parts of a star cluster, is one of the consequences of two-body relaxation and of the evolution toward energy equipartition in stellar systems. The characteristic timescale for this process, $T_{m s}$, is, for a population of massive stars with mass $m_{h}, T_{m s} \sim\left\langle(m\rangle / m_{h}\right) t_{\text {relax }}$ where $t_{\text {relax }}$ is the cluster relaxation time and $\langle m\rangle$ the mean mass of the stars.

However, a number of young clusters with ages substantially less than the time needed to produce the observed segregation by standard two-body relaxation shows a significant level of mass segregation (e.g. Hillenbrand 1997, Hillenbrand \& Hartmann 1998, Fischer et al. 1998, de Grijs et al. 2002, Sirianni et al. 2002, Gouliermis et al. 2004, Stolte et al. 2006). Several theoretical studies (e.g. Klessen 2001, Bonnell et al. 2001, Bonnell \& Bate 2006 but see also Krumholz et al. 2005, Krumholz \& Bonnell 2007) have suggested that massive stars would form preferentially in the center of star-forming regions and that the observed segregation in young clusters would be primordial.

Possible dynamical routes leading to early mass segregation in young star clusters have also been studied in McMillan, Vesperini \& Portegies Zwart (2007 and this volume) and Vesperini, McMillan \& Portegies Zwart (2008a in prep.).

Whatever the origin of the observed mass segregation in young clusters is, it is important to explore and understand the implications of initial mass segregation for the evolution of clusters. All the theoretical and numerical studies of the dynamical evolution of star clusters have adopted unsegregated initial conditions and nothing is known about the evolution of mass-segregated clusters. We have carried out a survey of $N$-body simulations to study the evolution of initially segregated clusters. We have explored the evolution of tidally truncated clusters located at different galactocentric distances and with different degrees of initial segregation and compared the evolution of the structure, stellar content and the lifetimes of segregated clusters with those of clusters with the same initial density profile but no initial mass segregation. We present here some of the results of our investigation; a complete description of all the simulations and results will appear in Vesperini, McMillan \& Portegies Zwart (2008b, in prep.). 


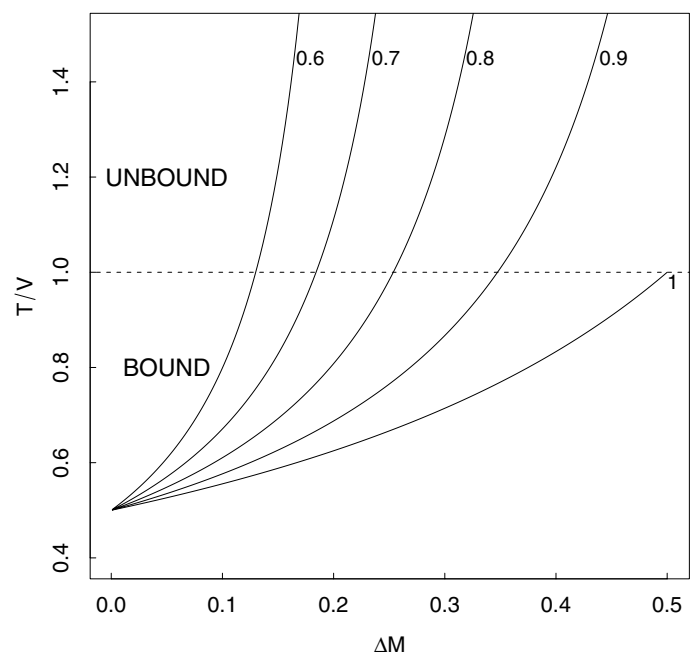

Figure 1. Virial ratio of a cluster with a Plummer density profile after the rapid mass loss of a $\Delta M$ fraction of its initial mass versus $\Delta M$. The density profile of the mass lost is assumed to be that of a Plummer model, but with a scale radius $a_{M L}$ equal to or less than the scale radius of the whole cluster, $a_{c l u s t e r}$. The number beside to each curve indicates the corresponding value of $a_{M L} / a_{\text {cluster }}$.

\section{Segregated cluster dynamical evolution: analytical estimates}

A number of studies (see e.g. Chernoff \& Shapiro 1987, Chernoff \& Weinberg 1990, Fukushige \& Heggie 1995) have shown that early mass loss due to stellar evolution can have a significant impact on the evolution of clusters: this early mass loss causes a cluster to expand and, for a low-concentration cluster, leads to the cluster complete and quick dissolution (see e.g. Vesperini \& Zepf 2003 for the possible implications of this early disruption on the properties of globular cluster systems). For initially segregated clusters, the mass lost due to the evolution of massive stars is removed preferentially from the cluster inner regions and the early expansion of the cluster is to be stronger and, potentially, more destructive than when the same amount of mass is lost in a nonsegregated cluster.

Fig. 1 shows the results of a semi-analytical calculation illustrating the augmented destructive effect of stellar mass loss in a mass-segregated cluster. Specifically, in this figure we plot the virial ratio, $T / V$, of an isolated cluster with an initial Plummer density profile, $\rho=\frac{3 M}{4 \pi a^{3}}\left(1+\frac{r^{2}}{a^{2}}\right)^{-5 / 2}$, after the impulsive loss of a fraction $\Delta M$ of the total mass. In order to mimic the preferential mass loss from the inner regions of a mass-segregated cluster, we have assumed that the density profile of the mass lost also follows a Plummer model, but with a scale radius $\left(a_{M L}\right)$ smaller than the scale radius of the cluster $\left(a_{\text {cluster }}\right)$. The curves in Fig. 1 show $T / V$ as a function of $\Delta M$ for different values of $a_{M L} / a_{\text {cluster }}$. For $a_{M L}=a_{\text {cluster }}$ one recovers the well-known result (Hills 1980) that a system becomes unbound for $\Delta M>0.5$. For clusters preferentially losing mass from the inner regions, $a_{M L}<a_{\text {cluster }}$, we see that a significantly smaller amount of mass loss, innocuous for an unsegregated clusters, can lead to dissolution of a segregated cluster.

This simple semi-analytical calculation underscores the potential crucial implications of initial mass segregation for the evolution of star clusters. 


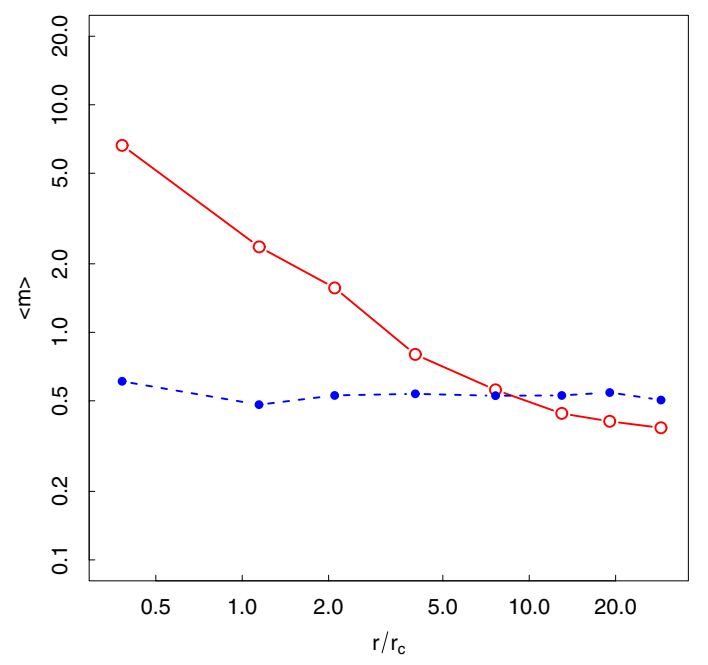

Figure 2. Initial radial profile of the mean mass of stars for the segregated (solid line) and the unsegregated clusters (dashed line) discussed in this paper.

\section{Segregated cluster dynamical evolution: $N$-body simulations}

In this section we discuss the results of a survey of $N$-body simulations aimed at comparing the evolution of initially segregated and unsegregated clusters. All the simulations have been carried out by the starlab package (Portegies Zwart et al. 2001; http: //www.manybody.org) We have followed the evolution of tidally truncated clusters with the same density profile, a Kroupa (Kroupa, Tout \& Gilmore 1993) stellar IMF, with and without initial mass segregation. Fig. 2 shows the initial radial profile of the star mean mass in the segregated and unsegregated clusters studied in our investigation.

We have performed a number of simulations comparing the evolution of segregated and unsegregated clusters at different galactocentric distances, $R_{g}$. For tidally truncated clusters with a fixed mass, the cluster global dynamical time and the amount of mass lost impulsively increase with the galactocentric distance, $R_{g}$ (we have also carried out a number of simulations of isolated clusters and clusters underfilling their tidal radius and explored the evolution for different levels of initial segregation; the results of these simulations will be presented in Vesperini, McMillan \& Portegies Zwart 2008b, in prep.)

Fig. 3 shows the dependence of the dissolution time, $T_{\text {diss }}$, (defined as the time when only 1 per cent of the initial mass is left in the cluster) on the clusters galactocentric distance for segregated and unsegregated clusters. All the unsegregated clusters survive the early mass loss due to stellar evolution and eventually dissolve as a result of the evaporation of stars due to two-body relaxation. On the other hand, early impulsive mass loss leads to the quick dissolution in a few dynamical times of all the initially segregated clusters. The only exception is the cluster closest to the galactic center for which the dynamical time is short enough that there is no significant impulsive mass loss; in this case the segregated cluster lifetime is similar to that of the corresponding unsegregated cluster.

The difference in the response to the same amount of impulsive mass loss in segregated and unsegregated clusters is further illustrated by the left panel of Fig. 4 which shows the time evolution of the core and half-mass radii for a segregated and an unsegregated cluster with the same initial density profile and located at the same galactocentric distance $\left(R_{g}=18 \mathrm{kpc}\right)$. Both clusters initially lose the same fraction of their initial mass and 


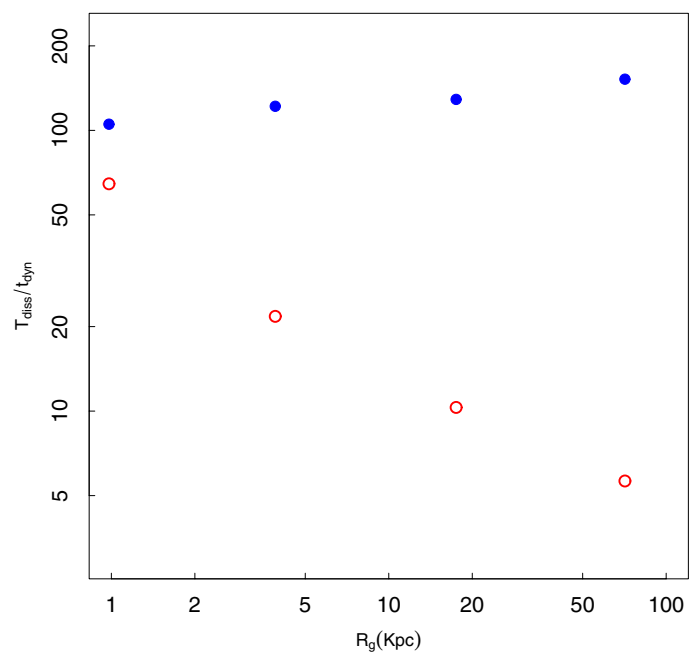

Figure 3. Ratio of the dissolution time, $T_{d i s s}$, to the dynamical time $t_{d y n}$, versus galactocentric distance for segregated (open dots) and unsegregated clusters (filled dots).
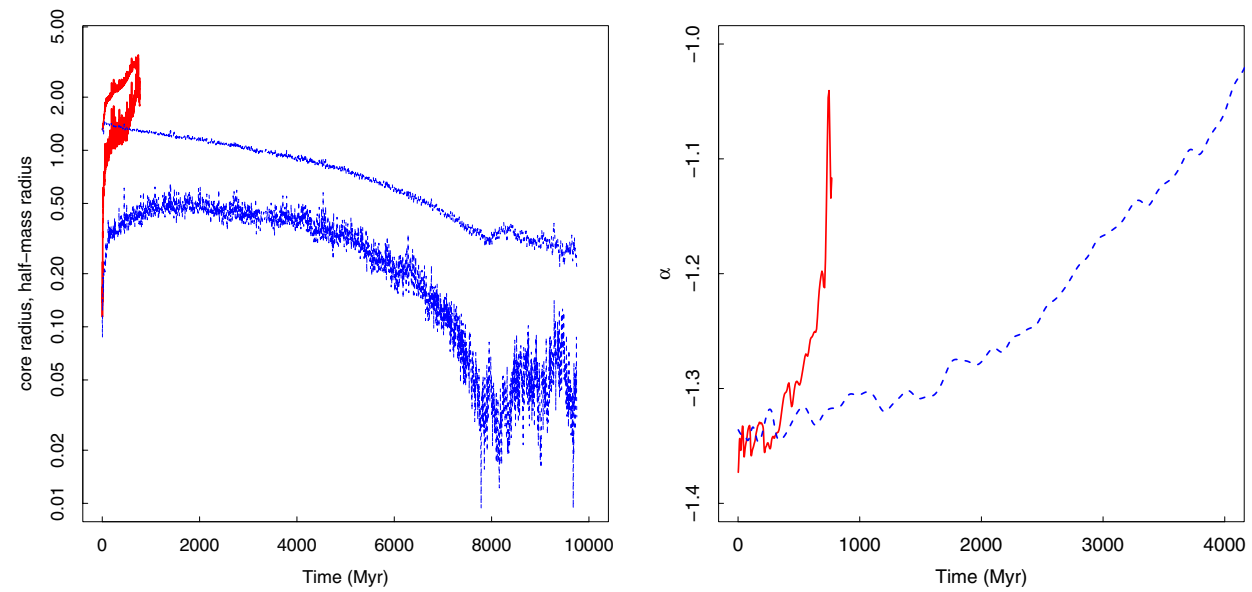

Figure 4. (Left panel) Time evolution of the core and the half-mass radii of a segregated (solid lines) and an unsegregated (dashed line) cluster. (Right panel) Time evolution of the slope of the mass function, $\alpha$, for stars with $0.1<m / m_{\odot}<0.5$ for a segregated (solid lines) and an unsegregated (dashed line) cluster.

expand in response to this mass loss; however, as anticipated by the semi-analytical calculations discussed in the previous section, the preferential removal of mass from the innermost regions in a segregated cluster, leads to a stronger expansion and to the cluster quick dissolution.

The right panel of Fig. 4 shows the time evolution of the slope of the mass function for low-mass stars $(0.1<m<0.5)$ and illustrates the implications of the different evolution of segregated and unsegregated clusters for the evolution of the clusters stellar content. Both segregated and unsegregated clusters preferentially lose low-mass stars but the 
timescale for the flattening of the mass function in the segregated cluster is significantly shorter than that of the unsegregated one.

\section{Conclusions}

We have presented the results of analytical calculations and numerical simulations aimed at exploring the differences in the evolution of initiallly segregated and unsegregated clusters. Our study shows that early mass loss due to stellar evolution leads to a stronger expansion of segregated clusters, a faster evolution toward a less concentrated structure and a faster dissolution. The evolution of the stellar content and, in particular, the preferential loss of low-mass stars also proceeds on a shorter timescale in segregated clusters.

A complete description of all the results of this investigation will be presented elsewhere (Vesperini, McMillan \& Portegies Zwart 2008b, in prep.)

Acknowledgments. This work was supported in part by NASA grants NNG04GL50G and NNX07AG95G, NSF grant AST-0708299, and by the Royal Netherlands Academy of Arts and Sciences (KNAW).

\section{References}

Bonnell, I. A., Clarke, C. J., Bate, M. R., \& Pringle, J. E. 2001, MNRAS, 324, 573

Bonnell, I. A. \& Bate, M. R., 2006, MNRAS, 370, 488

Chernoff D. \& Shapiro S., 1987, ApJ, 322, 113

Chernoff D. \& Weinberg M., 1990, ApJ, 351, 121

de Grijs, R., Gilmore, G. F., Johnson, R. A., \& Mackey, A. D. 2002, MNRAS, 331, 245

Fischer P., Pryor C., Murray S., Mateo M., \& Richtler T. 1998, AJ, 331, 592

Fukushige T. \& Heggie D., 1995, MNRAS, 276, 206

Gouliermis, D., Keller, S. C., Kontizas, M., Kontizas, E., \& Bellas-Velidis, I., 2004 , A\& A, 416, 137

Hillenbrand L. A., 1997, AJ, 113, 1733

Hillenbrand L. A. \& Hartmann L. E., 1998, ApJ, 331, 540

Hills J., 1980, ApJ, 235, 968

Klessen R., 2001, ApJ, 556, 837

Kroupa P., Tout C., \& Gilmore G., 1993, MNRAS, 262, 545

Krumholz M. R., Klein R. I. \& McKee C. F., 2005, Nature, 438, 332

Krumholz M. R. \& Bonnell I., 2007 astro-ph 0712.0828

McMillan S., Vesperini E., \& Portegies Zwart S., 2007, ApJ, 655, L45

Portegies Zwart, S. F., McMillan, S. L. W., Hut, P., \& Makino, J. 2001, MNRAS, 321, 199

Sirianni, M., Nota, A., De Marchi, G., Leitherer, C., \& Clampin, M. 2002, ApJ, 579, 275

Stolte, A., Brandner, W., Brandl, B., \& Zinnecker, H. 2006, AJ, 132, 253

Vesperini, E. \& Zepf, S., 2003, ApJ, 587, L97 